\section{Scaling the Information Load of Occupations: Preliminary Findings of the Fit Between Individual Capacities and Environmental Demands}

\section{Richard F. Haase}

University at Albany, State University of New York

\section{Joaquim Armando G. A. Ferreira}

Eduardo I. R. Santos

Universidade de.Coimbra, Portugal

Gina M. Aguayo

Melissa M. Fallon

University at Albany, State University of New York

Person-Environment (P-E) fit models provide a conceptually powerful way to think about career development, vocational choice, and occupational success. The work reported here focuses on yet another pair of P-E criteria: self-reported individual capacity for information processing (the ability to tolerate information overload from a variety of stimulus sources), and the corresponding demand characteristics for information processing of the occupational environment. To achieve the aims of this project, the authors have borrowed from the literature on information processing, anthropology, and human factors to define the information load context of the occupational environment. The authors have constructed a P-E congruence scheme for five domains of information processing: information load, interpersonal load, change load, activity structure, and time structure, and employed the methods of psychophysics to quantify occupational environments across these domains. The results of this preliminary work, replicated across two cultures, are presented here.

Keywords: information load, information processing capacity, magnitude estimation scaling, Person-Environment fit

Correspondence concerning this article should be addressed to Richard F. Haase, ED 220, Department of Educational and Counseling Psychology, University at Albany, State University of New York, 1400 Washington Avenue, Albany, NY 12222; e-mail: rfhremote@aol.com.

JOURNAL OF CAREER ASSESSMENT, Vol. 16 No. 2, May 2008 156-176 DOI: $10.1177 / 1069072707313184$

(C) 2008 Sage Publications
Since the beginning of the last century, one of the major theoretical perspectives in vocational psychology has been the Person-Environment (P-E) fit model (see for example, Savickas, 2000). As with any conceptual framework, this model has evolved historically and today is much more than a simple trait-and-factor theory; even different schools of the P-E fit theory have emerged (e.g., Holland, 1997; Lofquist \& Dawis, 1969). It is well known that P-E fit models provide a conceptually and empirically useful way to think about career development, vocational choice, and occupational success, broadly defined. In this study we advanced and tested a methodological technique for assessing yet another aspect of the P-E fit idea that taps the interface between self-reported individual differences in capacity for cognitive information processing and the informational demands inherent across differing occupations.

Within the P-E fit literature, the most prominent model is the scheme due to Holland $(1959,1997)$, whose hexagonal model of person and career types has dominated the field of vocational psychology for more than four decades (see, for example, Savickas, 1999). Although there appears to be some argument as to whether a circle or hexagon is the proper geometry to describe the model (Prediger, 1992; Holland \& G. D. Gottredson, 1992; Tracey \& Rounds, 1995), the validity of the idea of P-E fit as a conceptual scheme is firmly established in the both the science and practice of vocational psychology and career development.

The match between personality type (e.g., realistic, investigative, artistic, social, enterprising, and conventional [RIASEC]) and corresponding environmental personality type as defined by Holland (1959) is only one way in which P-E theory can be used to define congruence, assuming that congruence in turn should lead to vocational longevity, satisfaction, and success (Assouline \& Meir, 1988; Edwards \& Rothbard, 1999; Furnham, 2001). Other P-E fit schemes have been proposed that focus on the match between needs and occupational reinforcements (Lofquist \& Dawis, 1969), cognitive abilities and cognitive demands (L. S. Gottfredson, 2003), values and environmental rewards (Super, 1957), and personal resources and environmental demands (Holland, 1997), and more recently the match between persons and persons (Hartung, 2005)

In the work.we report here we focused on yet another P-E fit pair of criteria: individual capacity for information processing and its match to concomitant environmental demands for information processing. To accomplish our aims, we have borrowed concepts from anthropology and from the literature of information processing to define the concept of polychronicity at the individual level. We have also borrowed ideas from human factors, ergonomics, and cognitive psychology to deduce the implications of the interface between the information processing capacities of the individual and the demand characteristics of the environment. To these ends we have constructed a matching scheme for five types of information demands: information load, interpersonal load, change load, activity structure, and time structure. After a brief introduction of the main ideas that define the notion of information load in environments, and the capacities of individuals to tolerate these loads, we present the results of our attempt to quantify these constructs. We 
employed the methods of psychophysics - namely, magnitude estimation and category scaling (Stevens, 1975) - to quantify these constructs for both individuals and for the work environments they might occupy. As part of the quantification of these personal resources and environmental demands, we assessed their validity across two cultures. This work represents a prelirninary test of the potential utility of these ideas. Reasoning by analogy with marketing research, the initial testing of a concept or "an idea that is on its way to becoming a marketing strategy" (Dillon, Madden, \& Firtle, 1987, p. 608), is called a "proof of concept" and is conducted to establish the marketing potential of the product. The preliminary study that we report here is in the spirit of this "proof of concept" notion. There appears to be sufficient validity to the ideas entailed herein to warrant reporting our early results and to expanding on it in future research.

\section{POLYCHRONICITY}

The fundamental concept that underlies this study is that of polychronicity The seminal ideas that define the notion of polychronicity are attributable to the anthropologist E. T. Hall $(1959,1966)$ who documented the inherent differences across cultures in the way in which they handle time and space. Interaction distance (Hall called it proxemics) varies drastically between cultures. For example, the typical interaction distance between individuals in many South American and Mediterranean cultures is approximately 18 inches, whereas the typical North American or North European interaction distance is approximately 36 inches. North Americans in interactions with South Americans often report considerable negative arousal during the interaction, typically not understanding the source of their discomfort. They report their interaction partner as "pushy" and "aggressive" and as constantly "crowding" them during their conversations. Conversely, the South Americans report similar degrees of negative arousal, and attribute it to their interaction partner's "cold" and "aloof" style. It is easy to see how these subtle cultural differences can place unusual, even intolerable, environmental demands on the parties to the interaction, who are often unaware of the reason for their discomfort. Hall $(1959,1966)$ also dealt with the issue of cultural differences in the way that people structure time and how they structure activities that happen within a framework of time. Germany, for example is said to be a "two minute" culture, in which failures to meet on-time expectations can be perceived as a serious social transgression. It is said in North American universities that if the professor fails to show up for a scheduled class within $10 \mathrm{~min}$ of the appointed hour, the students are no longer obligated to wait-a $10 \mathrm{~min}$ culture. Latin and Mediterranean cultures are said to have much less "precise" or less rigid expectations for the handling of time and all that it implies in the context of interpersonal relations. Many Northern European cultures are said to have expectations for time management that are considerably more stringent than the American norm.

The point of this brief introduction to polychronicity is merely to suggest that "mismatches" can occur between individuals and environments in terms of handling information from two different sources (people and things) with both sources varying across both space and time. ${ }^{1}$ Information overload arising from one, or some combination, of these facets can often be a negatively arousing and unpleasant experience for the participants. The fact that such differences have been so clearly documented across cultures suggests that the personal and cognitive resources necessary to cope with different stimulus demands in the realms of information, time, and space may well be an individual differences variable that can be quantified. Once quantified, such individual differences might be matched to environmental demand characteristics of occupations that vary along similar dimensions.

\section{The Study of Stimulus Overload}

In the 1950s, 1960s, and 1970s, the perception of, and response to, conditions of stimulus overload as an aspect of individual differences was studied extensively in experimental, social, and clinical psychology (Bellak, 1975; Broadbent, 1971 G. A. Miller, 1959; J. G. Miller, 1961; Millord \& Perry, 1977, Wohlwill, 1974). The results of this long line of research have found practical application in many ergonomic situations (e.g., aviation psychology; Wiener \& Nagel, 1988); the evidence clearly reveals that individuals vary significantly in their sensitivity to, and ability to cope with, stimulus overloaded conditions. It is reasonably well established that individuals have limits on the amount of information they can process in any fixed period of time, with the classic index being about $7( \pm 2)$ bits of information (G. A. Miller, 1959). In the clinical domain of processing complex social information the task is more difficult and the estimated value of the transmission index is even smaller, at about 2 to 3 bits of information (Bieri et al., 1966). Contemporary cognitive psychology, in the study of parallel and serial processing (Pashler, 1994, Triesman \& Gelade, 1980), has extended our empirical and theoretical understanding of how information overload can impede performance, and how individual differences in information processing capacity can predict responses to stimulus overloaded environments (Logan, 2002; Pashler, 1994).

By analogy we take this significant body of literature on human information processing under stimulus overloaded conditions to be applicable to the kind of situational demands that may well differ from workplace to workplace and from occupation to occupation. We argue here that occupations could be differentiated by the extent to which they place differing stimulus demands on the practitioners of that occupation. Some occupations may be hugely loaded with demands to process visual, intellectual, nonpersonal information (e.g., air traffic controller), whereas other occupations may be largely in the domain of high interpersonal demands (e.g., psychotherapist, customer service representative). 
Some occupations may have heavy demands associated with the mastery of rapidly changing information (e.g., stock broker), whereas other occupations place serious demands on the occupants to structure the timing and sequence of events (e.g., construction supervisor). The psychological research that suggests this variation in informational demands across situations led us to the speculation that occupations may posses a variety of differing characteristics that could be quantified in terms of the stimulus demands that an occupation may place on its practitioners. The possibility that a match between person and an occupation across the dimensions of stimulus demands we define here (information load interpersonal load, change load, time structure, and activity structure) is suggested in the vocational literature. Holland's notions of the resulting success, satisfaction, and longevity that derive from a match between person and environment has been extensively documented. L. S. Gottfredson's (1980) validity study of Holland's hexagonal system verified that occupational environments can be differentiated on the basis of the demands inherent in that occupation. For example, low level social jobs were found to differ considerably in their demand for coping with interpersonal information from high level social occupations. Prediger's (1982) landmark study of the factor structure of occupations also supports the notion that the dimensions of data versus ideas and people versus things are important in the differing demands contained in any given occupation across these two broad domains. Similar ideas of P-E match across differing demand conditions is also seen in the Internet-based O*NET (U.S. Department of Labor, 2007), in which the occupational skills that can be matched to occupational requirements are a prominent feature of self-evaluation of occupational interest and choice.

In the work reported here, we focused on facets of the demand characteristics of occupational environments that are somewhat different than that which is included in other methods such as $\mathrm{O}^{*} \mathrm{NET}$ or Holland's self-directed search. Our focus is not on skills or abilities, but more on the individual differences in temperament and emotional tone that may govern the extent to which an individual can tolerate or cope with stimulus overload emanating from several sources. We return to a more detailed discussion of these five factors of the stimulus and information load of occupations following an introduction of a similar classificatory system that has been used to understand individual differences in the capacity to tolerate differing degrees and different types of stimulus and information overload.

\section{The Polychronicity Index (PCI)}

As noted in previous paragraphs, Hall $(1959,1966)$ introduced the idea of polychronicity as a method of differentiating cultures by the degree to which they place different situational demands on people within that culture. Concomitantly, Hall defined the difference between polychronic and monochronic cultures by the fashion in which members of that culture deal with time and space. Haase and his colleagues (Haase, 1986; Haase, Lee, \& Banks, 1979) adapted Hall's original ideas of polychronicity and monochronicity to the level of individual differences. Combining the work of Hall with the previously cited evidence emerging from the study of stimulus overload and parallel processing, we reasoned that individuals could be said to differ in their capacity for coping with stimulus overloaded situations; moreover, we classified both individuals and occupations in light of five constructs. For each of these constructs, we are primarily interested in the degree to which an individual has (or lacks) the capacity to cope with stimulation that naturally varies as a function of the work environment. In short, we conceptualize these constructs in terms of P-E fit indices. The five constructs that define this P-E fit classification system include (1) information overload, (2) interpersonal overload, (3) change overload, (4) time structure, and (5) activity structure. The PCI (Haase, 1986; Haase et al., 1979) was devised to evaluate individual differences on these five dimensions. The 25 -item version of the scale, 5 items per factor, was found to have internal consistency reliabilities ranging from .60 to .76 , with a 2 -month test-retest reliability of .67. Principal components analysis with varimax rotation confirmed the proposed 5-factor structure. The convergent validity of the full-scale polychronicity construct is supported by statistically significant relationships to flexibility $(+)$, tolerance of ambiguity $(+)$, sensation seeking $(+)$, authoritarianism $(-)$, neuroticism, $(+)$ and extraversion (-), and cognitive complexity (Haase et al., 1979). At the same time, the full scale score is unrelated to age, socioeconomic status, or reported stimulus characteristics of the early home environment. In a direct test of the relationship between polychronicity and capacity for information overload, two multiple cue, stimulus overloaded laboratory analogues revealed that polychronic individuals are significantly better able to cope with information overloaded situations than are self-reported monochromic individuals (Haase, 1986; Dumont \& Vamos, 1975). There is also evidence that the subscales of the PCI, as used in the present work, are differentially valid for different tasks (Haase, 1986).

In the paragraphs that follow, we provide a more careful definition of the constructs and integrate that definition with an attempt to scale a variety of occupations as to their capacity to produce stimulus overload on the same five dimensions. In particular, we will describe a methodology for evaluating the degree to which individuals and occupations might be matched on five dimensions of stimulus overload.

\section{Scaling the Social Consensus-Magnitude Estimation Scaling}

The methods of psychophysical scaling have been used to quantify peoples' subjective perceptions about the psychological magnitude of stimuli that can also be related to the intensity of an underlying physical stimulus. Perceptions of brightness, loudness, texture, warmth, and so forth have been extensively studied (Stevens, 
$1966,1975)$ and it is reasonably well accepted that the perceived psychological magnitude of a stimulus $(\Psi)$ is a power function of it's underlying physical metric $(\varphi)$,

$$
\Psi=k \varphi^{\beta}
$$

in which $k$ is an arbitrary constant of scaling, and $\beta$ is the exponent of the power function. The important point for our present purpose is that what Stevens (1975), and many others, have shown is that even though many elusive psychological constructs have no discernable underlying physical dimension, direct magnitude estimation can still be used to quantify constructs such as attitudes, preferences, and other psychological perceptions. Moreover, the cumulative evidence of nearly six decades has shown that these "scales of opinion" do indeed form ratio scales with a true zero point, order, and equal intervals that are characteristic of ratio scales. A vast number of other perceptual objects have been scaled by the methods of psychophysics (Gescheider, 1997; Stevens, 1975). Some classic examples of the use of magnitude estimation scaling to quantify psychological perceptions that have no underlying corresponding physical metric include the scaling of the stress value of life events (Holmes \& Rahe, 1967), the prestige of occupations (Kuennapas \& Wikstroem, 1963), and the seriousness of offenses (Sellin \& Wolfgang, 1964).

Our intention in this project was to quantify for occupations the same five information load conditions that we have previously defined for individuals. We have reasoned that occupations can be seen as possessing different degrees of demand characteristics of information load, interpersonal load, change load, time structure, and activity structure. For example, the realistic occupation of bank teller probably contains a relatively high degree of time and activity structure, but a relatively low demand for information processing, coping with interpersonal overload, or assimilating significant change overload. Contrast this with the investigative occupation of college professor, which more than likely possess very high levels of information and change loads, modest levels of environmental demand for interpersonal interactions, and relatively low demands for time and activity structure. If one can quantify the levels of these five demand characteristics across work environments, and if one can measure the capacity for tolerating the same five loads in the individual, then it would be possible to form a P-E fit of the profile of these characteristics. With such a profile it may be possible to better understand or predict vocational choice, longevity, satisfaction, and success.

In the remainder of this manuscript, we describe the methods that were employed to quantify the informational demand loads of 24 occupations chosen to represent the six RIASEC categories of Holland's (1997) hexagonal model. The results of this scaling process are presented along with an initial test of the replicability of the scaling across two cultural groups.

\section{METHOD}

We employed direct magnitude estimation scaling methods (Gescheider, 1997; Lodge, 1984; Stevens, 1975) to quantify the degree to which 24 occupations, four from each of the six Holland RIASEC categories and chosen to represent different levels of occupation, are perceived to possess information load, interpersonal load, change load, time structure, and activity structure.

\section{Participants}

A total of 77 university students (25 Portuguese and 52 American) served as participants in this study. The sample consisted of $84 \%$ females and $16 \%$ males, and ranged in age from 18 to 42 (mean age $=22, S D=4.1$ ). The largest group identified themselves as Caucasian American (48\%) followed by Portuguese (22\%); the remaining $30 \%$ of the respondents spread across five other ethnic/country-of-origin identifications (Italian, British, African American, Asian American, Hispanic, and French). Students completed the demographic questionnaires and the magnitude estimation scaling tasks individually or in small groups. Although it could be argued that the scale values obtained might be more trustworthy had we used employed workers rather than students, we chose students as a matter of convenience and also because there is some conviction that occupational judgments of this type are stereotypical and learned early in life (L. S. Gottfreson, 1996; Holland, 1997).

\section{Stimulus Materials}

A brief demographic questionnaire requested information on the respondents' age, gender, educational background, occupational plans, mother and father's education, and mother and father's occupations. Each respondent was also asked to list, in order of importance, up to eight occupations that they had identified and planned to enter. The first expressed occupational choice was selected for each participant and a single letter high-point code was assigned according the classification system of Holland (1997).

The magnitude estimation scaling tasks were performed for each of the five information load conditions on each of the 24 occupations chosen to represent the categories of Holland's hexagonal arrangement of occupations-realistic, investigative, artistic, social, enterprising, and conventional. Prior to beginning the first magnitude estimation task, the participant was given instructions on how to conduct the experiment by way of a simple example of magnitude estimation. The instructions were standard definitions and instructions (Lodge, 1984; Stevens, 1975) and appeared as follows:

Magnitude estimation scaling is a method that allows you to make comparative judgments about the value of objects. For example, if you were asked to judge the usefulness of modes 
of transportation, and automobile is arbitrarily assigned 50 units of usefulness, you might make the following judgments:

$$
\text { Automobile } \underline{50} \quad \text { Airplane } \underline{25} \quad \text { Bicycle } \underline{10}
$$

in which you are judging an airplane to be $1 / 2$ the value of automobile, and automobile as 5 times more valuable than bicycle. You can use any numbers you like to record your judgments to reflect the relative value of the objects.

As another example, you might judge the relative importance of a library, the internet, and other people as sources of information about travel. If library is arbitrarily assigned 50 units of value, you might make the following judgments:

$$
\text { Library } 50 \text { Internet } \underline{100} \text { Other People } 5
$$

in which you are judging the internet to be 2 times more informative than a library, other people to be 20 times less informative than the internet, and the a library to be 10 times more informative than other people."

Following these instructions, the participants were asked to apply magnitude estimation ratings to each of the 24 occupation names. The participant repeated the task five times, each time judging the extent to which each occupation name was considered to have relative amounts of information load, interpersonal load, change load, activity structure, and time structure. For each information load rating task, a standard occupation (e.g., engineer) was assigned a value of 50 and all the remaining 23 occupation names were judged comparatively when considered against this standard. In addition, all respondents completed a measure of polychronicity that measures an individual's estimate of their capacity for tolerating overload across the five conditions of information, interpersonal, load, time, and activity.

\section{RESULTS}

Means and standard deviations of the magnitude estimated scale value for each of the 24 occupation names have been presented in Table 1 . It was clear that the participants in this experiment were able to discriminate between occupations with respect to their perceived levels of the load or demand that attaches to each occupation across the five areas of information, interpersonal, change, time, and activity. As seen in Table 1, the scale values range from a low of 18 to a high of 101 and center around a value of approximately $50 .^{2}$ There is sufficient variability across occupations and across information loads to justify further investigation of the variability among the ratings. In particular, we speculated that there may be systematic differences of the scale values across countries (Portugal vs. United States), across occupational type (RIASEC type), between information load conditions, and as a combination of the three factors. The validity of the ratings might be investigated as a function of expected differences in scale values that might be observed for the main and interaction effects of these three conditions.
Table 1

Magnitude Estimated Scale Values of 24 Occupations and

\begin{tabular}{|c|c|c|c|c|c|}
\hline & $\begin{array}{l}\text { Information } \\
\text { Load }\end{array}$ & $\begin{array}{l}\text { Interpersonal } \\
\text { Load }\end{array}$ & $\begin{array}{l}\text { Change } \\
\text { Load }\end{array}$ & $\begin{array}{c}\text { Activity } \\
\text { Structure }\end{array}$ & $\begin{array}{c}\text { Time } \\
\text { Structure }\end{array}$ \\
\hline \multicolumn{6}{|l|}{ Realistic Occupations } \\
\hline Construction inspector & $41(20)$ & $55(30)$ & $46(30)$ & $51(29)$ & $57(33)$ \\
\hline Electrician & $32(20)$ & $46(17)$ & $34(23)$ & $47(62)$ & $53(34)$ \\
\hline Plumber & $18(13)$ & $50(00)$ & $30(22)$ & $47(63)$ & $51(33)$ \\
\hline Tool designer & $39(26)$ & $.28(20)$ & $40(30)$ & $44(63)$ & $46(28)$ \\
\hline \multicolumn{6}{|l|}{ Investigative occupations } \\
\hline Biologist & $71(26)$ & $39(30)$ & $55(22)$ & $58(58)$ & $58(29)$ \\
\hline Chemist & $72(28)$ & $38(33)$ & $50(00)$ & $60(61)$ & $62(34)$ \\
\hline Physicist & $73(28)$ & $40(30)$ & $56(20)$ & $56(61)$ & $58(28)$ \\
\hline Research scientist & $78(34)$ & $40(41)$ & $68(30)$ & $59(63)$ & $57(35)$ \\
\hline \multicolumn{6}{|l|}{$\begin{array}{l}\text { Kesearch sclentist } \\
\text { Artistic occupations }\end{array}$} \\
\hline Art dealer & $34(21)$ & $67(42)$ & $48(41)$ & $40(39)$ & $46(25)$ \\
\hline Author & $43(24)$ & $30(27)$ & $34(23)$ & $43(64)$ & $43(40)$ \\
\hline Commercial artist & $38(22)$ & $69(89)$ & $53(40)$ & $39(25)$ & $47(28)$ \\
\hline Musician & $34(24)$ & 48.(39) & $43(40)$ & $40(59)$ & $50(00)$ \\
\hline \multicolumn{6}{|l|}{$\begin{array}{l}\text { Musician } \\
\text { Social occupations }\end{array}$} \\
\hline Marriage counselor & $52(27)$ & $89(50)$ & $52(34)$ & $49(35)$ & $64(33)$ \\
\hline School superintendent & $53(27)$ & $84(42)$ & $59(41)$ & $57(47)$ & $66(35)$ \\
\hline Speech therapist & $53(21)$ & $86(44)$ & $53(28)$ & $51(32)$ & $68(31)$ \\
\hline Teacher & $50(00)$ & $101(57)$ & $61(32)$ & $67(35)$ & $82(34)$ \\
\hline \multicolumn{6}{|l|}{ Enterprising occupations } \\
\hline Business executive & $62(29)$ & $78(43)$ & $68(42)$ & $55(25)$ & $66(35)$ \\
\hline Hotel manager & $37(22)$ & $83(45)$ & $45(41)$ & 49 (32) & $56(34)$ \\
\hline Real estate salesman & $35(20)$ & $79(44)$ & $50(40)$ & $47(24)$ & $54(26)$ \\
\hline Stock and Bond Salesman & $58(38)$ & $77(48)$ & $72(46)$ & $50(00)$ & $62(42)$ \\
\hline \multicolumn{6}{|l|}{ Conventional occupations } \\
\hline Bank teller & $32(20)$ & $68(31)$ & $31(23)$ & $53(64)$ & $59(37)$ \\
\hline Bookkeeper & $32 \cdot(24)$ & 37. (34) & $27(22)$ & $50(65)$ & $58(44)$ \\
\hline Financial analyst & $62(31)$ & $61(55)$ & $72(46)$ & $47(28)$ & $61(30)$ \\
\hline Statistician & $59(25)$ & $31(24)$ & $43(28)$ & $54(59)$ & $53(26)$ \\
\hline
\end{tabular}

Five Information Loads

Note. Scale values are the means of 26 Portuguese and 52 U.S. respondents. Standard deviations are in parentheses. A standard deviation of 0 indicates the standard stimulus.

\section{Three Factor Split Plot Factorial Analyses of the Magnitude Estimated Scale Values}

The 24 occupations studied here were each rated across five informational demand conditions, which were further crossed with six Holland occupational types. Every respondent rated each occupation in these 30 combinations. These two factors comprise a $5 \times 6$ factorial arrangement with repeated measures on each factor. Crossing these two repeated measures factors with a two-level between subjects factor of Portuguese and American respondents created a $2 \times$ $5 \times 6$ split plot factorial design (Kirk, 1992; Maxwell \& Delaney, 2004). 
The main effects from a repeated measures analysis can be statistically salient, but equally often uninteresting because the mean structure being tested is aggregated across all other conditions, which obscures the more interesting differences found in interactions. Nonetheless, Portuguese and American respondents did not differ in their mean magnitude estimated value averaged across 24 occupations, six Holland types, and five informational demand conditions (Portuguese mean = 52.33, American mean $=55.33 ; F(1,75)=.038, p=.847)$. The mean magnitude estimated scale values for the five levels of information load, aggregated across respondents and Holland types, did show significant differences. As expected, the five load types were seen to differ in terms of their average demand characteristics (Pillai's Trace $=.295$, approximate $F(4,72)=7.54, p<.001) .{ }^{3}$ Also, significant differences were found across Holland types, aggregated across informational demands and respondents $(F(5,71)=36.58, p<.001)$. Although statistically significant, these mean differences are too gross to be of any real interest. What are far more informative from these analyses are the two-way and three-way interactions that were also statistically significant. The differences between scale values for Holland occupations by information loads are displayed in Figure 1.

The three-way (Country $\times$ Load Type $\times$ Holland Type) interaction proved to be statistically significant (Pillai's Trace $=.402, F(20,42)=1.88, p<.033$ ) and of a sizeable magnitude (multivariate partial $\eta^{2}=.402$ ); about $40 \%$ of the partialled variance of the estimated scale values is accounted for by the triple interaction. The three-factor interaction has been graphically displayed in Figure 2. The twoway Holland Type $\times$ Country interaction was also statistically significant (Pillai's Trace $=.349, F(5,71)=7.62, p<.001)$. Its interpretation, however, is largely captured in some of the simple main effects observed within the three-way interaction, and a graph of the two-way interaction is not presented here.

Visual inspection of the graphs presented in Figure 2 reveal that the underlying source of the interaction between Portuguese and American judges lies primarily in the differences they observe when rating the artistic occupations. A MANOVA comparing the vector of estimated scale values within only the artistic occupations between Portuguese and American judges was statistically significant (Pillai's Trace $=.343$, approximate $F(5,71)=7.42, p<.001$ ). Univariate follow-up $F$ tests revealed that the differences between national judge groups on the artistic occupations rested mainly in the differences between Portuguese and American judges on information load (mean difference $=49$ vs. $32 ; F(1,76)=$ $19.44, p<.001$ ), on interpersonal load (mean difference $=66$ vs. $47 ; F(1,76)=$ $5.21, p<.025$ ), and on change load (mean difference $=53$ vs. $40 ; F(1,76)=3.21$, $p<.077)$. No significant differences between Portuguese and American judges were observed on both activity structure and time structure. In each of the instances in which differences were observed, Portuguese judges endowed the artistic occupations as a group with considerably more environmental demands for information processing, interpersonal interactions, and the necessity to cope with rapidly changing environmental work conditions. At a later point we offer some speculations about cultural differences that may help to explain these
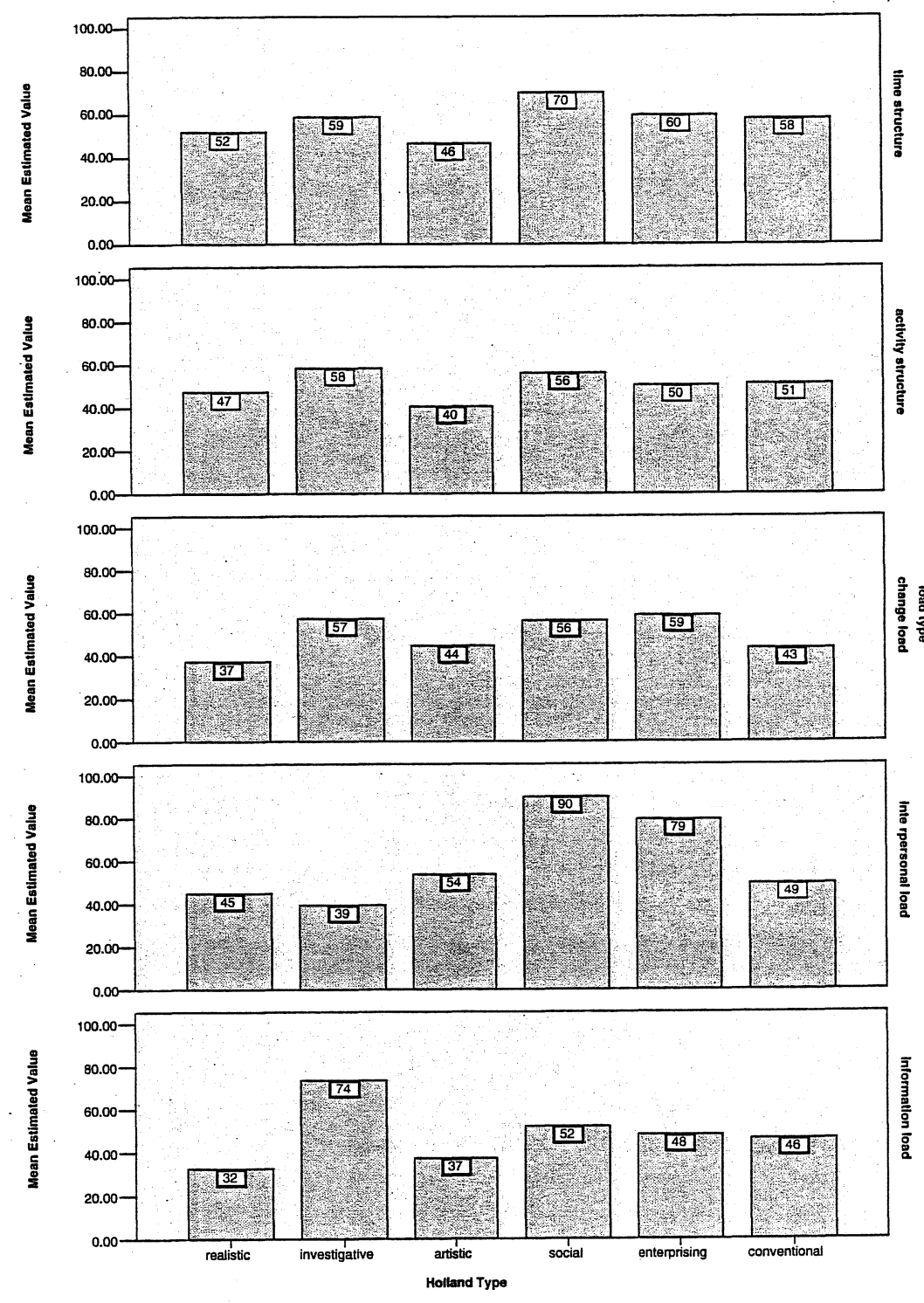

Figure 1. Differences in Scaled Load Demands Across Six Holland Types 


\section{Occupation by Load Interaction}

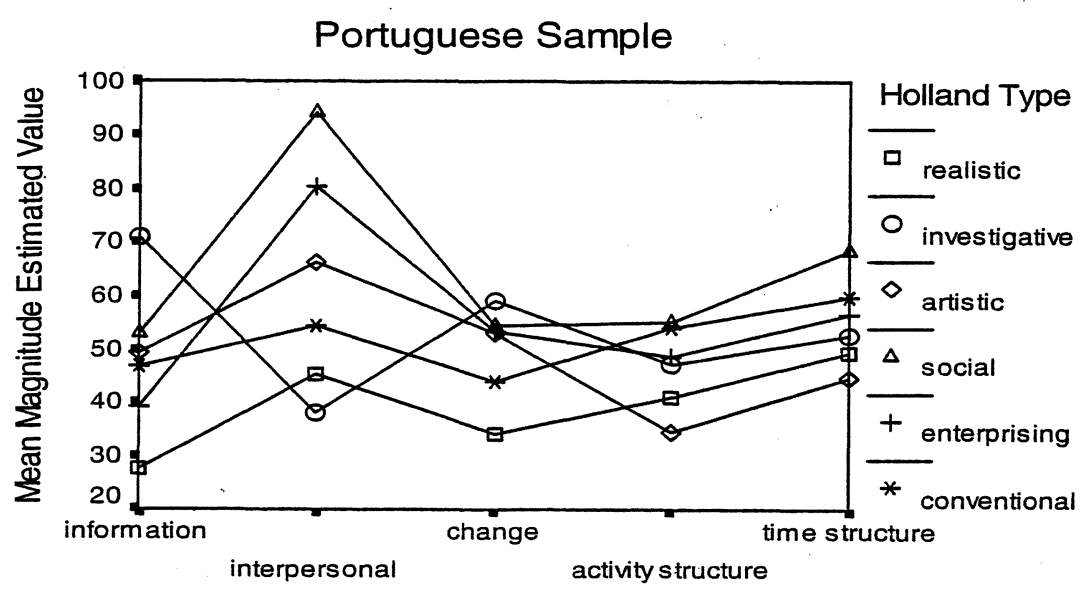

Type of Load

\section{Occupation by Load Interaction}

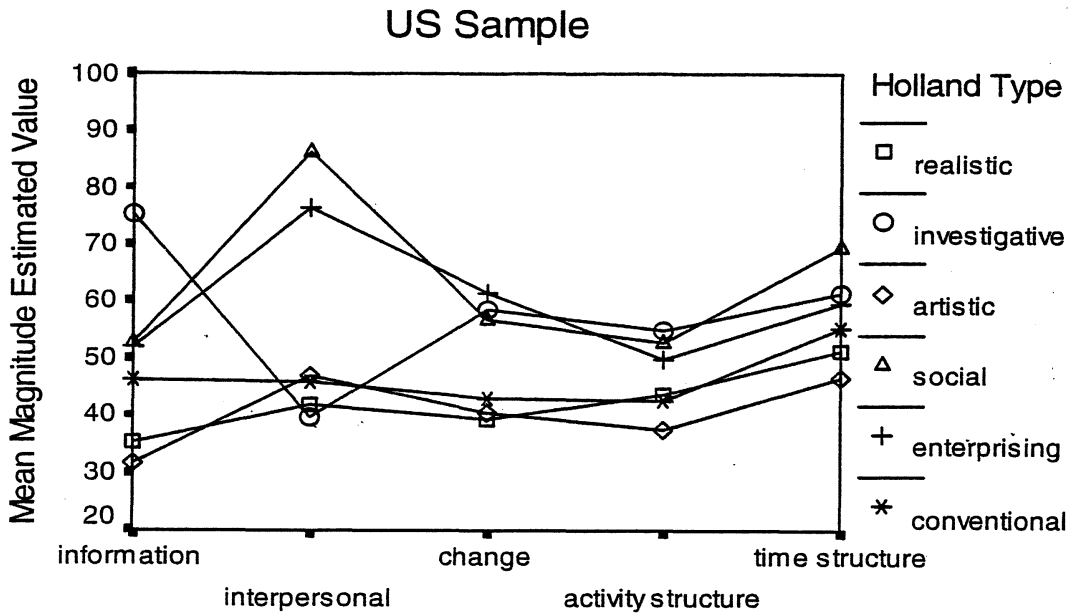

Type of Load

Figure 2. Three-Way Interaction Between Country, Holland Type, and Information Load Type observations. Although the graphs of Figure 2 suggest that the Portuguese and American judges may have perceived differences in the conventional occupations as well, a two-group, one-way MANOVA on the five load values of the conventional occupations showed no difference between countries (Pillai's Trace $=$ $.081, F(5,72)=1.27, p=.284)$.

Beyond the Artistic occupations, therefore, the profiles of the magnitude estimated scale values are substantially similar across both Portuguese and American judges. We conclude from these data that the environmental demands for information processing in most occupations, with the exception of the artistic occupations, are perceived similarly in the two countries. The degree of similarity in the magnitude estimated scale values across the two countries is also revealed in the correlation between the 120 separate occupation-by-load values estimated by Portuguese and American judges: $r(120)=.80$.

\section{Polychronicity and the Discriminant Validity of} the Estimated Scale Values

In the introduction to this article we emphasized that the ultimate goal of establishing scaled values of environmental loads of occupations may rest in the ability to match a person to an environment-the P-E fit hypothesis. A person with a high capacity for information processing and a low capacity for interpersonal stimuli would find an occupational setting with opposite environmental demands, marriage counselor for example, to be difficult to tolerate for long periods of time. Hence, the idea of having a method for "scaling individuals" in terms of their capacity for tolerating information, interpersonal, and change load conditions, as well as their characteristic preferences for time and activity structure, would be necessary and beneficial in establishing a P-E fit on these constructs. To assess these individual preferences, we collected individual responses to the PCI (Haase et al., 1979) from the participants in this study. As a matter of general interest, we were curious as to whether Portuguese and American respondents might show differences, perhaps culturally imbedded, in their profiles of their capacity for information load processing. But much more importantly, it was our intention to test the divergent validity of the magnitude estimated scale values given to the $120(24 \times 5$, Occupation $\times$ Load $)$ ratings. That is, it is important to demonstrate that although individual judges, both Portuguese and American, can be reliably differentiated from one another in terms of their information processing capacities, and be shown to have considerable individual differences, it must be established that the magnitude estimated scale values of the rated occupations provided by these judges are independent of their own persona status on the five constructs of interest. To have trustworthy scale values for the occupations and their demand loads, such values should be shown to be "objective" in the sense that they are free of bias due to an individuals standing on the capacity for tolerating certain forms of environment-induced informational demands. In short, one's capacity for tolerating information overload should not 
be related to one's judgments about the degree of informational demand in any given occupation.

To test this proposition, we chose to study the averaged scale values of the five information load conditions across the six Holland occupational types-30 averaged scale values in all. A multivariate multiple regression analysis (Finn, 1974; Johnson \& Wichern, 1992) predicting the observed variation in the 30 scaled values (see Table 1) as a function of the judges individual polychronicity profiles (i.e., PCI scales 1 through 5) was fitted to the data. No significant multivariate correlation could be found between the 30 scaled values of the occupations and the individual Polychronicity profiles of the 77 judges (Pillai's Trace $=1.91$, approximate $F(150,230)=.95, p<.63)$. In addition to this whole model test, no individual partialled PCI scale (information, interpersonal, change, activity, time) was found to be significantly related to the set of 30 occupation scale values (All $F$ tests on 30 and 42 degrees of freedom were less than 1.3 , and $p$ values ranged from .29 to .99). Thus we are reasonably confident that the magnitude estimated scale values presented in Table 1 are free of bias that might be introduced by the individual predilections of the judges; the Portuguese and American judges were able to be objective while performing the magnitude estimation scaling task.

\section{Cross Cultural Differences in Estimated Scale Profiles}

Although there was no relationship between individual capacities for information load processing and the scale values obtained by magnitude estimation, we questioned whether Portuguese and American judges would show any systematic differences in their Polychronicity profiles. A test of the differences in the 5-scale PCI profiles between Portuguese and American judges in this study was performed by a multivariate profile analysis (a split plot design with country of origin being the between-subjects factor and the five scales of the PCI being the repeated measures factor). The test of the country-by-PCI scale interaction in this design is a test of the profile equivalence across the two groups of judges (Tabachnick \& Fidell, 2001). We found a significant country-by-PCI interaction (Pillai's Trace $=.199$, multivariate $F(4,72)=4.47, p<.003$ ), which suggests that the profiles of the Portuguese and American judges are not equivalent across all five PCI scales. The interaction reveals that the average American and Portuguese judge are relatively similar in terms of their preferences for activity and time structure (a surprise to us), but appear to differ in their reported capacity for handling information load, interpersonal load, and change load. In these three instances, the Americans report significantly greater tolerances for information load $(17.3$ vs. $15.4, p<.05)$, interpersonal load $(17.8$ vs. $14.5, p<.05)$ and change load $(16.9$ vs. $14.6, p<.01)$ than their Portuguese counterparts. Statistical significance was tested by simple main effect contrasts between judge groups within each level of the five PCI scales; the simple differences between udges were statistically significant at $p<.05$ for the information, interpersonal, and change scales, but not statistically significant for time and activity structure.

\section{Some Initial Tests of Concurrent Validity}

Although the major purpose of the present study was to quantify the information loads in 24 occupations, we did attempt to collect some indicators from the respondents that might be used to perform initial tests of concurrent validity. As part of the demographic questionnaire, all respondents were asked to list their college major as well as up to eight occupations they were considering, ranked in order of their importance. Because virtually all the respondents were psychology or education majors there is insufficient variability in the college major category to be of much value. However, 67 of the 75 respondents expressed specific and fairly diverse occupational interests for which we assigned a single letter highpoint Holland code. The high-point codes from this sample represented five of the six Holland types, lacking only the conventional category. Given each respondent's information processing profile obtained from the PCI, we were able to match that profile to the six RIASEC load profiles obtained from the average magnitude estimated scale values. We then computed five correlations for each respondent assessing the degree of "match" between their stated preferences for tolerating the five informational loads measured by the PCI and the five demand characteristics of the occupational types. For each respondent, we assigned a profile high-point code to the occupational profile (RIASEC) that most closely matched their own profile of capacities for information loads. The correlations between these two profiles could (and did) range from +1.0 to -1.0 . A high positive correlation indicates a "good" profile match. We cross-tabulated this profile match high-point code (RIASEC) with the expressed interest high-point code (RIASEC) across respondents and found that that the high point codes for 21 of 67 respondents, $31 \%$, matched exactly. Chance alone would predict 1 in 6 correct classifications $(17 \%)$. The chi-square goodness of fit test applied to the $6 \times 5$ classification revealed a statistically significant relationship between high-point code derived from the P-E profile match and the high-point code obtained from the respondent's expressed occupational interest (Cramer's $V=.343, \chi^{2}(20)=$ $31.57, p=.048$ ). Although it is not an especially rigorous test of congruence (a single sample for calibration and cross-validation), the data are sufficiently strong to be encouraging of more careful validity studies. Moreover, the congruence coefficient related to this $31 \%$ correct classification rate is well within the range of those reported in recent meta-analyses (Tsabari, Tziner \& Meir, 2005). Whether this degree of congruence would ultimately translate into career success, satisfaction, and longevity is a question yet to be answered.

Finally, as an initial attempt to provide some construct validity for the idea of a P-E fit based on information processing capacity and demands, we examined the relationship between salary for the 24 occupations and the magnitude estimated scale value of those occupations for each of the five information load dimensions. We reasoned that increasing degrees of environmental demand should be tracked by increasingly greater requirements for entry-level education and for higher salaries (G. D. Gottfredson, Holland, \& Ogawa, 1982). We fitted a regression 
model of salary to the magnitude estimated scale values across the 24 occupations. We fitted a separate model for each of the five information load demand categories. Significant proportions of variability in salary are accounted for by the demand characteristics of information load $\left(r^{2}=.41, p<.001\right)$ and change load $\left(r^{2}=.33, p<.003\right)$. No significant relationships were found for interpersonal load, time structure, or activity structure. The fitted regression model for information load reveals that for every one unit increase in estimated information load of an occupation, salary would increase by $\$ 558$. Similarly, for change load, an increment of $\$ 613$ is observed for every unit increase in the demand to cope with rapidly changing information in the occupation. Both Portuguese and American cultures obviously place considerable monetary value on occupations that necessitate dealing with increasingly demanding information loads and rapidly changing information conditions. Conversely, increasing interpersonal occupationa demands (say, from plumber to psychotherapist) are virtually unrelated to monetary compensation $\left(r^{2}=.021, p>.500\right)$. Occupations scaled to have zero demand characteristics for coping with interpersonal overload are empirically estimated to decrease by $\$ 93$ for every one unit increase in the scaled interpersonal demand characteristics of the occupation. ${ }^{5}$ Apparently, society is far more willing to compensate those who deal with high informational load occupations than it is to compensate comparable workloads in the interpersonal domain.

\section{DISCUSSION}

We consider the study reported here to be a "proof of concept" evaluation in the sense that this term is used in marketing research (Dillon et al., 1987). We hoped to be able to demonstrate that we could quantify the demand characteristics of occupations in such a way as to provide the possibility for assessing P-E fit for individuals who can be also ordered on their self-reported capacity for processing information along the same dimensions. To this end, we employed the methods of direct numeric magnitude estimation to arrive at scale values that have true ratio properties (Lodge, 1984; Stevens, 1975) which in turn would allow for direct ratio comparisons of occupations in terms of the extent to which a given occupation may possess some level of environmental demand for information processing, interpersonal interaction, capacity for tolerating change, and the imperatives for activity and time structure. We feel that the objectives of this work have been achieved.

A modest degree of construct validity was observed in these data, in that certain of our early expectations have been borne out. For example, the perceived environmental demands for information processing corresponds to our notions of the extent to which occupations in different Holland codes (RIASEC) may place different demands on the individuals in that occupation. Hence we speculated that investigative occupations would have inherently larger demands for processing of information than would, say, realistic occupations. Such is the case; the average scaled value of the investigative occupations was approximately 74 , as compared to a value of approximately 33 for realistic occupations. Hence it is legitimate to say that the information processing demands of investigative occupations are more than twice that of realistic occupations, twice that of artistic occupations (37), 1.4 times larger than the informational demand of social occupations (53), 1.5 times more demanding than enterprising occupations (48), and 1.6 times more demanding than conventional occupations (46). Similarly, interpersonal demands are seen to be uniformly high in social occupations $(90)$ which are more than twice as interpersonally demanding as investigative occupations (39), and nearly equal (ratio of $1.1: 1$ ) to the interpersonal demand characteristics of enterprising occupations (79). Such comparisons can also be made for the factors of change load, time structure, and activity structure. It is our contention that a high degree of face validity of the overall scaling task is similarly reflected in the differential scale values of Table 1 . Realistic occupations appear to be relatively undemanding in terms of all five demand characteristics scaled in this study, a fact that is consistent with our intuition. Social occupations are seen to have exceptionally high interpersonal demand characteristics, as well as relatively high demands for time structure - $a$ fact to be expected because counselors, teachers, speech therapists, and so forth, are all highly dependent on scheduling and appointments because the work involves scheduled service delivery.

As a practical matter, our intent in this "proof of concept" study was to establish that the information processing demand characteristics of different occupations could be reliably estimated. The purpose in doing so is to provide an "informational profile" for occupations against which the comparable "capacity profile" of an individual can be compared. We speculated that matching profiles would increase the probability that a person in that occupation would experience success, satisfaction, and longevity, compared to a person for whom the occupational choice is a "mismatched" profile. Although we cannot measure that kind of congruence-to-satisfaction link with the data of this study, the fact that expressed interest and the information load profile match are contingently, and significantly, related is encouraging. Future research must eventually attend to the ultimate issue of job-related satisfaction, success, and longevity as a matter of establishing construct validity for the ideas tested in this study. In addition, the fact that salary levels of these 24 occupations can be so clearly predicted from information-load and change-load demand characteristics is an encouraging finding in terms of the validity of the magnitude estimated scale values. Clearly, society (as reflected through the eyes of our 75 judges) values the ability to process information and to cope with change-and they are willing to pay for it For those in the social service industries, the data are not encouraging-there was virtually no connection between compensation and the characteristic demands of such occupations (e.g., interpersonal load, time structure). Neither Portuguese nor American judges appear to place compensatory monetary value on increasingly demanding social work environments. We are intrigued by the 
fact that this finding is independent of country - the global economy has caught up with all of us.

Finally, we are intrigued by the differences we observed between Portuguese and American judges with respect to the absolute levels of the environmental demand characteristics. From the perspective of an American (RFH) who has limited knowledge and experience of Portuguese culture, language, and customs, the Portuguese seem to place considerable importance on artistic endeavors, and artistic interests and activities seem to be uniformly infused into the whole of the social and occupational fabric of the country. Their long history of emphasizing art and architecture is obviously valued in the current occupational structure of the country. Contrast that to the United States, where artistic persons are often marginalized and thought to be just a little bit "off," and clearly are on the lower rungs of the economic ladder (with the exception of a very few successful artists). From the perspective of the Portuguese (JAF and EJR), there are indeed differences in which art and aesthetic appreciations are embedded in Portuguese culture and society. It is common for example for many "public persons" (e.g. politicians, scientists) to have parallel "careers" in writing, painting, or other artistic endeavors. And the public and private physical spaces of Portugal often reflect architectural historic, and artistic dimensions. These apparent and intriguing differences will require further investigation to understand more fully the impact of culture and society on the perception and understanding of occupations and their demands.

We set out to demonstrate our ability to quantify the information load that might be characteristic of several domains of occupations. Our goal was to demonstrate that occupations can be quantified in the extent to which they possess differing demand characteristics - that is, differing degrees of the need to process information, cope with interpersonal challenges, deal with continually changing work conditions, and structure both work activities and the time from within which work is accomplished. Moreover, we attempted to examine the match between self-reported tolerances for information loads and the demand characteristics of chosen occupations on the same dimensions. We are currently in the process of additional experimentation to solidify the ideas and provide further evidence of the potential utility of this scheme for assessing another form of P-E fit and to examine cultural differences as they might influence the perception of occupations and work environments.

\section{NOTES}

1. The concepts that underlie the polychronic-monochronic distinction are not unlike the two-dimensional classification system of Prediger (1982) - data versus ideas, and people versus things. These connections are discussed more fully in later paragraphs.

2. Setting the numeric value of the standard for judgment (e.g., engineer $=50$ ) will tend to constrain the mean scale value near that number. The allocation of numeric standard is still a matter of some controversy in the scaling literature (Gescheider, 1997; Lodge, 1984; Stevens, 1975).

3. Pillai's Trace was employed as the multivariate test statistic for any test involving a repeated measures factor. The multivariate test does not require the restrictive assumption of compound symmetry of the within cells variance-covariance matrices and is more trustworthy if such an assumption is not met as required by the univariate mixed model $F$ tests. Discussion of these conditions is found in Maxwell and Delaney (2004).

4. The typical standard deviation for both Portuguese and American samples is around 3.3. Hence a 2-poin mean difference is an effect size of approximately two thirds of a standard deviation, and a 3-point difference is a full standard deviation difference. Cohen (1988) defines such effects as "large." All three differences between judge groups are statistically significant by follow-up tests of simple main effects (Kirk, 1992).

5. Technically, the confidence interval of the regression line of salary on interpersonal load contains 0 . The regression does not reach any acceptable level of statistical significance and the $-\$ 93$ slope is very likely to be sampling error.

\section{REFERENCES}

Assouline, M., \& Meir, E. (1988). Meta-analysis of the relationship between congruence and wellbeing measures. Joumal of Vocational Behavior, 28, 319-332.

Bellak, L. (1975). Overload. New York: Human Sciences Press.

Bieri, J Atkins, A L., Briar S. Leaman, R. L., Miller, H., \& Tripodi, T. (1966). Clinical and social judgment: The discrimination of behavioral information. New York: Wiley.

Broadbent, D. E. (1971). Decision and stress. New York: Academic Press.

Cohen, J. (1988). Statistical power analysis for the behavioral sciences. Mahwah, NJ: Lawrence Erlbaum

Dillon, W. R., Madden, T. J., \& Firtle, N. H. (1987). Marketing research in a marketing environment. St. Louis, MO: Times Mirror/Mosby College Publishing.

Dumont, F. R., \& Vamos, P. (1975). Multimodal stimulus processing and polychronicity. Unpublished manuscript, Montreal, Quebec, Canada: McGill University.

Edwards, J., \& Rothbard, N. (1999). Work and family stress and well-being: An examination of person-environment fit in the work and family domains. Organizational Behaviour and Human Decision Processes, 77, 85-129.

Finn, J. D. (1974). A general model for multivariate analysis. New York: Holt, Rinehart \& Winston. Finn, J. D. (1974). A general model for multivariate analysis. New York: Holt, Rinehart \& Winston. urnham, A. (2001). Vocational preference and P-O fit: Reflections on
tional choice. Applied Psychology: An International Joumal, 50, 5-29.

tional choice. Applied Psychology: An International Joumal, 50, 5-29.
Gescheider, G. (1997). Psychophysics: The fundamentals. Mahwah, NJ: Lawrence Erlbaum.
Gottfredson, G. D., Holland, J. L., \& Ogawa, D. K. (1982). Dictionary of Holland occupational

Gottfredson, G. D., Holland, J. L., \& Ogawa, D. K. (1982). Dictionary of Holland occupationa codes. Palo Alto, CA: Consulting Psychologists Press.

Gottfredson, L. S. (1980). Construct validity of Holland's occupational typology in terms of prestige, census, Deparment of Labor, and other classification systems. Joumal of Applied Psychology, 65, 697-714.

Gottfredson, L. S. (1996). Gottfredson's theory of circumspection and compromise. In D. Brown and L. Brooks, (Eds.), Career choice and development (3rd ed., pp. 179-232). San Francisco: Jossey-Bass Publishers.

Gottfredson, L. S. (2003). The challenge and promise of cognitive career assessment. Journal of Career Assessment, 11, 115-135.

Haase, R. F. (1986). Polychronicity and strength of the nervous system as predictors of information overload. In J. Strelau, F. H. Farley, \& A. Gale (Eds.), The biological bases of personality and behavior: Psychophysiology, performance, and application. Washington, DC: Hemisphere.

Hase, R. F, Lee, D. Y, \& Banks, D. L. (1979). Cognitive correlates of polychronicity. Perceptud and Motor Skills, 49, 271-282.

Hall, E. T. (1959). The silent language. New York: Doubleday.

Hall, E. T. (1966). The hidden dimension. New York: Doubleday.

Hartung, P. J. (2005). Integrated career assessment and counseling: Mindsets, models, and methods. In W. B. Walsh \& M. L. Savickas (Eds.), Handbook of Vocational Psychology (3rd ed., pp. 371-395). Mahwah, NJ: Lawrence Erlbaum. 
Holland, J. L. (1959). A theory of vocational choice. Journal of Counseling Psychology, 6, 35-45. Holland, J. L. (1997). Making vocational choices: A theory of vocational personalities and work environments. Odessa, FL: Psychological Assessment Resources, Inc.

Holland, J. L., \& Gottfredson, G. D. (1992). Studies of the hexagonal model: An evaluation (or, the perils of stalking the perfect hexagon). Journal of Vocational Behavior, 40, 158-170.

Holmes, T. H., \& Rahe, R. H. (1967). The social readjustment rating scale. Journal of Psychosomatic Research, 11, 213-218.

Johnson, R. A., \& Wichern, D. W. (1992). Applied multivariate statistical analysis. Englewood Cliffs, NJ: Prentice Hall.

Kirk, R. E. (1992). Experimental design. Monterey, CA: Brooks/Cole

Kuennapas, T., \& Wikstroem, I. (1963). Measurement of occupational preferences: A comparison of scaling methods. Perceptual and Motor Skills, 17, 611-624.

Lodge, M. (1984). Magnitude scaling. Beverly Hills, CA: Sage.

Logan, G. D. (2002). Parallel and serial processing. In H. Pashler and J. Wixted (Eds.), Stevens' handbook of experimental psychology (3rd ed.). New York: John Wiley \& Sons.

Maxwell, S. E., \& Delaney, H. (2004). Designing experiments and analyzing data. Mahwah, NJ: Lawrence Erlbaum.

Lofquist, L. H., \& Dawis, R. V. (1969). Adjustment to work. New York: Appleton-Century-Crofts.

Miller, G. A. (1959). The magical number seven, plus or minus two: Some limits on the capacity for processing information. Psychological Review, 63, 81-97.

Miller, J. G. (1961). Sensory overloading. In B. E. Flaherty (Ed.), Psychophysiological aspects of space flight. New York: Columbia University Press.

Millord, J. T., \& Perry, R. P. (1977). A methodological study of overload. The Journal of General Psychology, 97, 131-137.

Pashler, H. (1994). Dual-task interference in simple tasks: Data and theory. Psychological Bulletin, $116,20-244$

Prediger, D. J. (1982). Dimensions underlying Holland's hexagon: Missing link between interests and occupations? Joumal of Vocational Behavior, 22, 259-287.

Prediger, D. J. (1992). Who claims Holland's hexagon is perfect? Joumal of Vocational Behavior $40,210-219$.

Savickas, M. L. (Ed.). (1999). [Special issue]. Journal of Vocational Behavior, 55(1).

Savickas, M. L. (2000). Person-environment fit: Theoretical meaning, conceptual models, and empirical measurement. Joumal of Vocational Behavior, 56, 145-146.

Sellin, T., \& Wolfgang, M. E. (1964). The measurement of delinquency. New York: Wiley.

Stevens, S. S. (1966). A metric for the social consensus. Science, 151, 530-541.

Stevens, S. S. (1975). Psychophysics. New York: John Wiley \& Sons.

Super, D. E. (1,957). The psychology of careers. New York: Harper and Row.

Tabachnick, B. G., \& Fidell, L. S. (2001). Using multivariate statistics. Needham Heights, MA Allyn \& Bacon.

Tracey, T. J. G., \& Rounds, J. (1995). The arbitrary nature of Holland's RIASEC types: A concentriccircles structure. Journal of Counseling Psychology, 42, 431-439.

Triesman, A., \& Gelade, G. (1980). A feature integration theory of attention. Cognitive Psychology, $12,97-136$.

Tsabari, O., Tziner, A., \& Meir, E. I. (2005). Analysis of the relationship between congruence and satisfaction. Joumal of Career Assessment, 13, 216-232.

U.S. Department of Labor. (2007). O*Net Online [Interactive Web application]. Retrieved December 21, 2007, from www.onetcenter.org

Wiener, E. L., \& Nagel, D. C. (1988). Human factors in aviation. New York: Academic Press.

Wohlwill, J. F. (1974). Human adaptation to levels of environmental stimulation. Human Ecology, $2,127-147$.

\section{College Students' Perspectives on Their Career Decision Making}

\author{
Shawn T. Bubany \\ Thomas S. Krieshok \\ Michael D. Black \\ Robyn A. McKay \\ University of Kansas
}

This mixed methods study examined how college student participants discussed their approach to making career decisions, with a focus on how their perspective may be consistent with various models of career decision making. Brief telephone interviews were conducted with 20 college students, and the narrative data were analyzed using qualitative methods informed by grounded theory and consensual qualitative research (COR). Based on themes generated by qualitative analysis, a ratings instrument was developed. The instrument was used by two individuals who dually served as auditors of the qualitative findings and as independent raters. Themes, as well as correlations and frequency data derived from the ratings, are discussed. It was found that the views of the participants, in terms of how they thought decisions should be made and how they were approaching their own decisions, were consistent with models of career decision making that include notions of interdependence, experience, intuition, and emotion.

Keywords: career decision-making models, mixed methods, intuition, interdependence, engagement

A number of scholars are questioning the rational orientation that has been dominant in the study of career decision making since the monumental work of Parsons in 1909 (e.g., Gelatt, 1989; Hartung \& Blustein, 2002; Krieshok, 1998, 2001; Mitchell, Levin, \& Krumboltz, 1999; Phillips, 1994, 1997). An issue at the forefront of the discussion concerns the roles played by reason and intuition in career decision-making models (Hartung \& Blustein, 2002). In a review of the

Shawn T. Bubany is now at Department of Psychology, University of Minnesota. The authors thank Craig Beeson, Selby Conrad, Sarah Sharma, Brian Sullivan, and Maggie Syme for their assistance with the qualitative analysis. This study is based on Shawn T. Bubany's master's thesis conducted under the direction o Thomas S. Krieshok at the University of Kansas. Correspondence concerning this article should be addressed to Thomas S. Krieshok, Department of Psychology and Research in Education, 1122 West Campus Road, Room 621, Lawrence, KS 66045; e-mail: tkrieshok@ku.edu.

JOURNAL OF CAREER ASSESSMENT, Vol. 16 No. 2, May 2008 177-197 DOI: $10.1177 / 1069072707313189$

(c) 2008 Sage Publications 